\title{
Quantitative phase imaging of live cells using fast Fourier phase microscopy
}

\author{
Niyom Lue, Wonshik Choi, Gabriel Popescu, Takahiro Ikeda, Ramachandra R. Dasari, \\ Kamran Badizadegan, and Michael S. Feld
}

\begin{abstract}
Using the decomposition of an image field in two spatial components that can be controllably shifted in phase with respect to each other, a new quantitative-phase microscope has been developed. The new instrument, referred to as the fast Fourier phase microscope (f-FPM), provides a factor of 100 higher acquisition rate compared with our previously reported Fourier phase microscope. The resulting quantitative-phase images are characterized by diffraction limited transverse resolution and path-length stability better than $2 \mathrm{~nm}$ at acquisition rates of 10 frames/s or more. These features make the f-FPM particularly appealing for investigating the structure and dynamics of live cells over a broad range of time scales. In addition, we demonstrate the possibility of examining subcellular structures by digitally processing the amplitude and phase information provided by the instrument. Thus we developed software that can emulate phase contrast and differential interference contrast microscopy images by numerically processing FPM images. This approach adds the flexibility of digitally varying the phase shift between the two interfering beams. The images obtained appear as if they were recorded by variable phase contrast or differential interference contrast microscopes that deliver an enhanced view to the subcellular structure when compared with the typical commercial microscope. (C) 2007 Optical Society of America OCIS codes: $180.3170,170.1530,170.0180$.
\end{abstract}

\section{Introduction}

Techniques such as phase contrast $^{1}$ and Nomarski/ differential interference contrast (DIC) microscopy ${ }^{2}$ provide a contrast of nearly invisible samples such as live cells by transforming the phase information into intensity distribution and thus revealing structural details of biological systems. However, the information obtained with these techniques about the phase shift associated with the illuminating field is only qualitative. Retrieving quantitative phase information from transparent objects with high accuracy and low noise allows for novel applications in the biological investigation of structure and dynamics.

N. Lue, W. Choi, G. Popescu (gpopescu@mit.edu), R. R. Dasari, K. Badizadegan, and M. S. Field are with the G. R. Harrison Spectroscopy Laboratory, Massachusetts Institute of Technology, Cambridge, Massachusetts 02139. N. Lue is also with the Department of Biomedical Engineering, University of Massachusetts, Multicampus, Boston, Massachusetts. T. Ikeda is with Hamamatsu Photonics K.K., 5000 Hirakuchi, Hamamatsu, Shizuoka 434-8601, Japan.

Received 28 June 2006; revised 3 October 2006; accepted 6 October 2006; posted 10 October 2006 (Doc. ID 72444); published 13 March 2007.

0003-6935/07/101836-07\$15.00/0

(C) 2007 Optical Society of America
Various point measurement techniques have been developed over the years for quantifying phase shifts at a given point through biological samples. This class of techniques can be described as an extension of optical coherence tomography $(\mathrm{OCT})^{3}$ to measurements of phase, phase dispersion, and birefringence associated with biological structures. Phase sensitive OCTtype measurements have also been performed for studying static cells, ${ }^{4}$ electrical activity in nerves, ${ }^{5,6}$ and spontaneous beating in cardiomyocytes. ${ }^{7}$ Using a harmonically related pair of wavelengths, $, 8,9$ and a phase-referenced interferometer, absolute phase and phase dispersion measurements have been performed with high stability. ${ }^{10}$

However, these methods rely on single point measurements, which for imaging purposes required raster scanning. Combining phase-shifting interferometry with Horn microscopy, digitally recorded interference microscopy with automatic phase shifting has been proposed as a new technique for quantitative biology. ${ }^{11-13}$ However, the method is susceptible to phase noise and may prevent accurate long-term dynamic studies. Based on the transportof-intensity equation, ${ }^{14,15}$ a quantitative-phase microscopy method was developed. This approach requires the displacement of the sample through the focus and extensive computations, which may limit its 


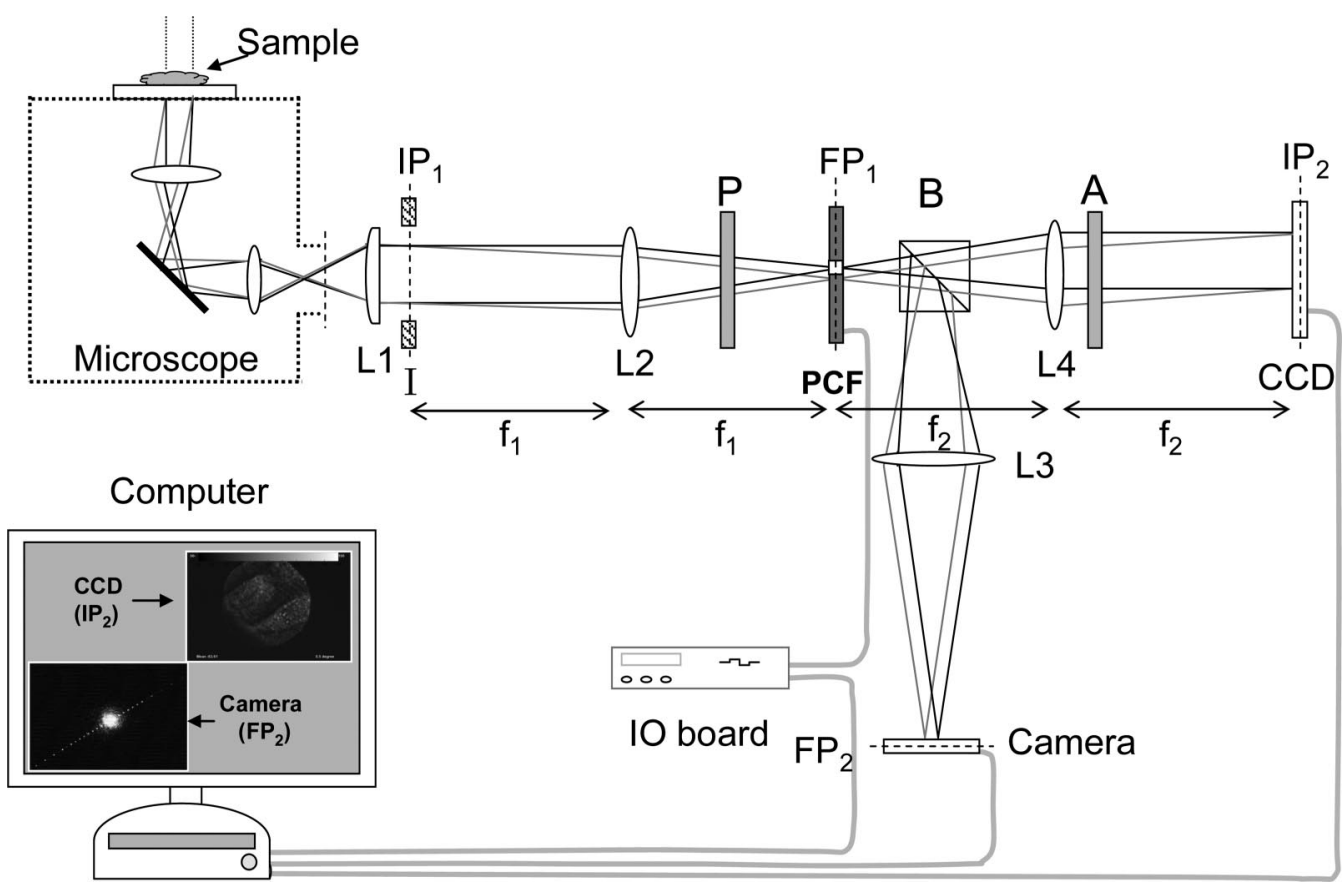

Fig. 1. Experimental setup.

applicability to dynamic biological studies. Digital holography is a phase-sensitive technique that combines traditional holography ${ }^{16}$ with digital recording and numerical calculations of Fresnel propagation. ${ }^{17}$ Recently, it has been used for imaging live cells and estimating their refractive indices. ${ }^{18-20}$

Our laboratory has focused on developing new full-field phase imaging techniques, which are suitable for spatially resolved investigation of biological structures. ${ }^{21-23}$ By combining the principles of phase contrast microscopy and phase-shifting interferometry, we have developed a new, extremely low-noise phase imaging microscope. ${ }^{21}$ The instrument, referred to as the Fourier phase microscope (FPM), produces very stable phase measurements at acquisition rates of up to 4 frames/min. However, many processes that take place at the cellular level, including cytoskeletal dynamics, cell membrane fluctuations, and organelle transport, occur at shorter time scales. Therefore a microscope that allows acquisition of fast full-field, high-stability quantitative phase images is highly desirable. We used the FPM principle in combination with a fast refresh rate liquid crystal modulator to obtain images at a rate of approximately $10 \mathrm{~Hz}$, which represents a factor of 100 improvement in speed with respect to our previous report. ${ }^{21,24}$ The new instrument is referred to as the fast Fourier phase microscope (f-FPM).

\section{Fast Fourier Phase Microscopy}

\section{A. Experimental Setup}

To obtain quantitative phase images with hightransverse resolution and low noise, we employed the principle of Fourier phase microscopy. In this technique, the optical field associated with a microscope image is decomposed into a high spatial frequency (ac) component and an average field (dc). We use a liquid crystal modulator to control the phase of the ac with respect to the dc component. Thus with this geometry we obtain the phase stability associated with common path interferometry, ${ }^{25,26}$ while using the principle of phase-shifting interferometry to retrieve quantitatively the phase of the sample field.

The experimental setup is shown in Fig. 1. The second harmonic of the cw Nd:YAG laser (CrytaLaser, special custom-built module; wavelength $\lambda=532 \mathrm{~nm}$, $500 \mathrm{~mW}$ ) is used as an illumination source for a typical inverted microscope (Axiovert 100, Carl Zeiss). To ensure spatial coherence and plane-wave illumination, the laser beam is coupled to a single-mode fiber, and the output is collimated. The light transmitted through the sample is collected by a $40 \times$ objective lens $(\mathrm{NA}=0.65)$. Lens $\mathrm{L} 1$ is set at the video port of the microscope to correct the beam divergence. An iris $\mathrm{I}$ is placed right at the image plane $\mathrm{IP}_{1}$ to control the size of the image. The image is transferred to the CCD using a $4 f$ system composed of lenses L2 $(f=1000 \mathrm{~mm})$ and $\mathrm{L} 4(f=700 \mathrm{~mm})$. At the Fourier plane (FP) we place the phase contrast filter [(PCF) custom made by Hamamatsu], which is made of nematic liquid crystal $2 \mathrm{~cm} \times 2 \mathrm{~cm}$ in aperture sandwiched between layers of indium tin oxide (ITO) electrodes. At the center of the PCF, a $150 \mu \mathrm{m}$ diameter circular portion of the ITO electrode is removed such that only the outer part of the PCF can have biasedphase modulation. The driving electric field is a square wave with $5 \mathrm{kHz}$ frequency, which is generated by an I/O board (National Instrument PCI-MIO-16XE-10, Austin, Texas). The Fourier lens L2 spatially decomposes the image field into its average component dc 
and a spatial varying or scattered field ac at the $\mathrm{FP}_{1}$. The position of the dc component is adjusted to overlap with the central pinhole of the PCF, such that only the scattered field ac undergoes phase shifting when voltage is applied. The dc and phase-shifted ac components interfere at the image plane of the $4 f$ system, and the resulting image is captured by a CCD camera (Hamamatsu C7770, $9.9 \mu \mathrm{m} /$ pixel, $640 \times 480$ pixels, $291 \mathrm{~Hz}$ ). For alignment purposes, we simultaneously image the FP. Using beam splitter B and lens L, the image of the PCF is captured by a video camera (Intercon Vision Products N50, Nortech, Minnesota). Since there is a continuous transition between the ac and the dc components, and the size of the PCF pinhole is finite, the aperture at $\mathrm{IP}_{1}$ was used to controllably select the dc component, i.e., the spatial low-frequency content of the image. If the dc spot size is too small relative to the nonmodulated central aperture of the PCF, part of the ac component can pass through the central hole, and therefore the reference beam itself shows strong spatial modulation. To eliminate this effect, we enlarged the spot size of the dc component in the FP by limiting the size of aperture I to $0.5 \mathrm{~cm}$ diameter, which produces a $50 \mu \mathrm{m}$ diffraction spot of the de beam. The drawback of this solution is that the field of view is now limited (smaller than the CCD chip). However, this is not a limitation in principle of this technique, as it can be resolved in the future by using a liquid crystal modulator with a smaller pinhole.

\section{B. Calibration of the Phase Contrast Filter}

The driving voltage across the ITOs determines the amount of phase retardation imposed on the ac component. To obtain a calibration curve phase versus voltage, we used a typical arrangement of electrooptic amplitude modulation, as follows. The PCF was illuminated by a large beam and placed between crossed polarizers ( $\mathrm{P}$ and $\mathrm{A})$. The PCF principal axes are $45^{\circ}$ with respect to the axis of polarizer $\mathrm{P}$, such that the liquid crystal modulator operates in amplitude mode. The I/O board is used to generate the driving electric field across the PCF and to digitally collect the transmitted intensity measured by an optical powermeter (Newport 1830, Irvine, California). The transmitted irradiance has the form

$$
I \propto \sin ^{2}[\Gamma(V) / 2],
$$

where $\Gamma$ is voltage-dependent phase retardation. The transmitted intensity with respect to the driving voltage is plotted in Fig. 2(a). Using an inverse trigonometric function, the wrapped phase $\Gamma$ is obtained

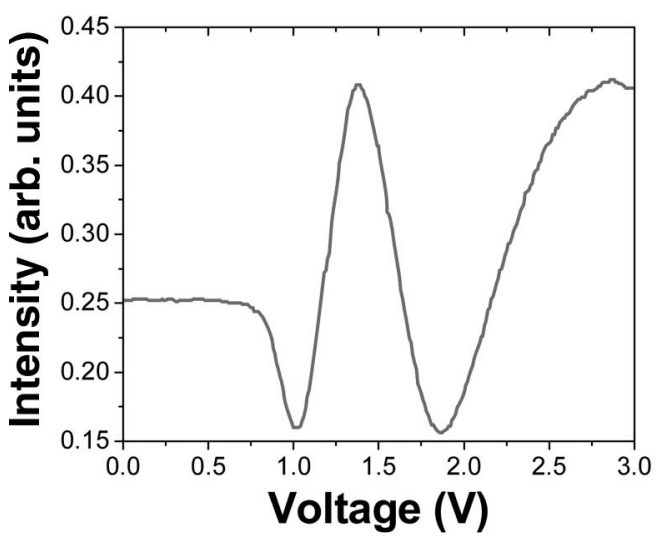

(a)

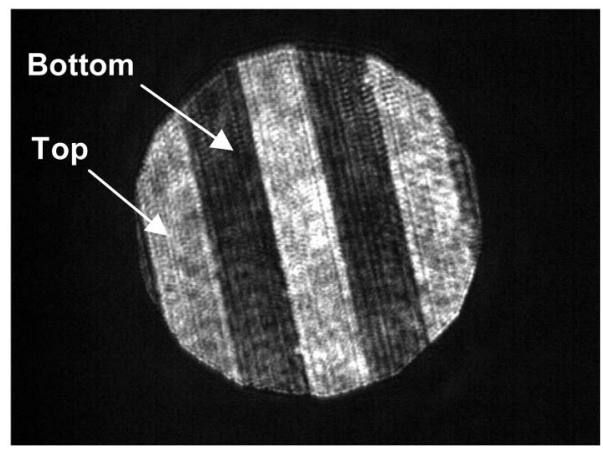

(c)

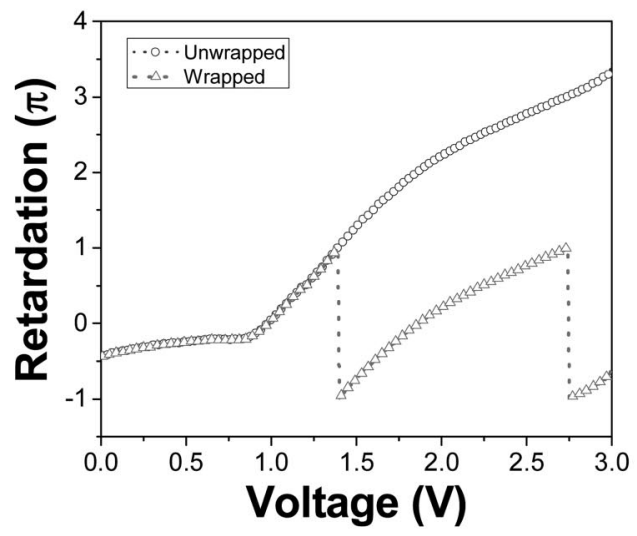

(b)

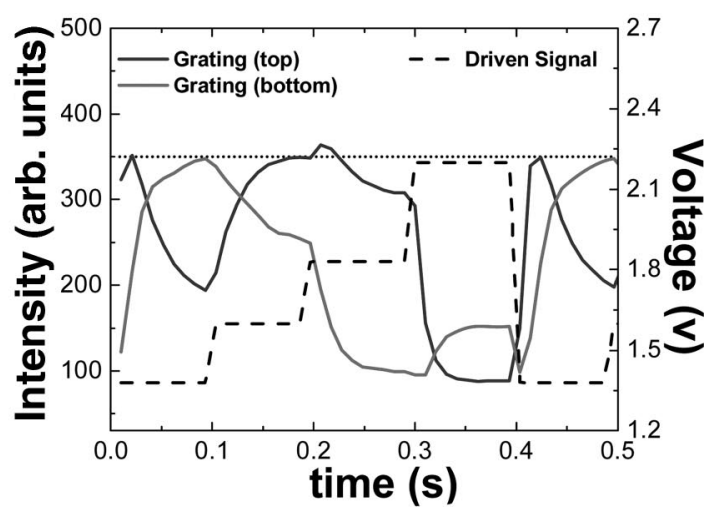

(d)

Fig. 2. (a) Intensity versus voltage associated with the PCF in amplitude mode. (b) Wrapped and unwrapped phase versus voltage calibration curve. (c) Phase grating during the PCF phase mode of operation. (d) Intensity variation versus step voltage for top and bottom areas on the phase grating in (c). The dotted line is a guide to the eye for the saturation level. 
[Fig. 2(b)]. Using a numerical procedure for unwrapping the phase, the final calibration curve, $\Gamma(V)$, was obtained, which is also shown in Fig. 2(b).

\section{Data Acquisition and Processing}

The four-frame phase-shifting interferometry algorithm is used to obtain the relative phase $\Delta \phi$ between the ac and the dc components. To achieve pure phase modulation on the ac field, the axis of polarizer $\mathrm{P}$ is set parallel to the principal axes of the PCF. The analyzer A is placed after the PCF with its axis parallel to $\mathrm{P}$. To take advantage of the linear portion of the calibration curve [Fig. 2(b)] we generated four interferograms corresponding to the phase shifts of the ac component by $\pi, 1.5 \pi, 2 \pi$, and $2.5 \pi$, corresponding, respectively, to $1.38,1.6,1.83$ and $2.2 \mathrm{~V}$. The spatially resolved phase difference between the ac and the dc fields can then be calculated as

$$
\Delta \phi(x, y)=\arctan \left(\frac{I_{1.5 \pi}(x, y)-I_{2.5 \pi}(x, y)}{I_{\pi}(x, y)-I_{2 \pi}(x, y)}\right),
$$

where $I_{\varphi}$ is the irradiance distribution of the interferogram obtained for phase shift $\varphi$.

To obtain the time response of the liquid crystal modulator and thus assess the achievable acquisition speed of the instrument, we monitored the intensity change across a phase grating image as a function of the driving voltage. Since the grating is a phase object, it appears transparent (i.e., shows no contrast) if no phase difference is applied between the ac and the dc components. However, the grooves of the grating become visible once voltage is applied on the PCF, as shown in Fig. 2(c). Figure 2(d) shows the variation of the irradiance in time associated with two neighboring grating grooves, as the voltage is stepped. To calibrate the response of the PCF, we set the time interval for individual phase steps long enough for the liquid crystal to reach the steady state. The analysis of the PCF response time yielded a value of less than $50 \mathrm{~ms}$. Therefore in our experiments we applied phase shifts at intervals of $100 \mathrm{~ms}$. The CCD acquires the image during the last $10 \mathrm{~ms}$ of each interval. As a result, we were able to acquire the phase images up to $10 \mathrm{~Hz}$. Using the ratio $\beta$ of the field amplitudes ac and $\mathrm{dc}$, the spatially dependent phase associated with the microscope image is obtained $a^{21}$

$$
\phi(x, y)=\arctan \left(\frac{\beta \sin [\Delta \phi(x, y)]}{1+\beta \cos [\Delta \phi(x, y)]}\right) .
$$

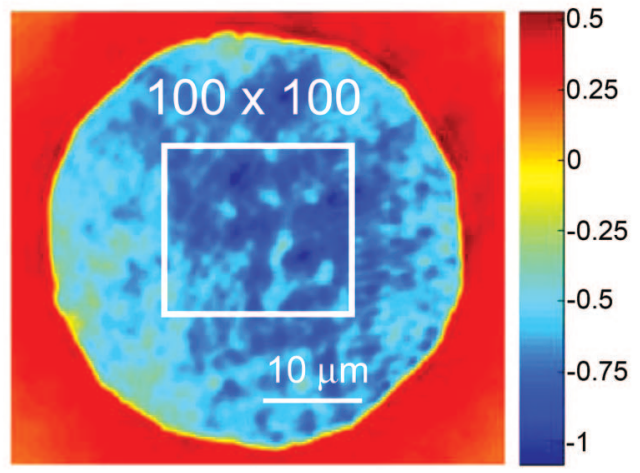

(a)

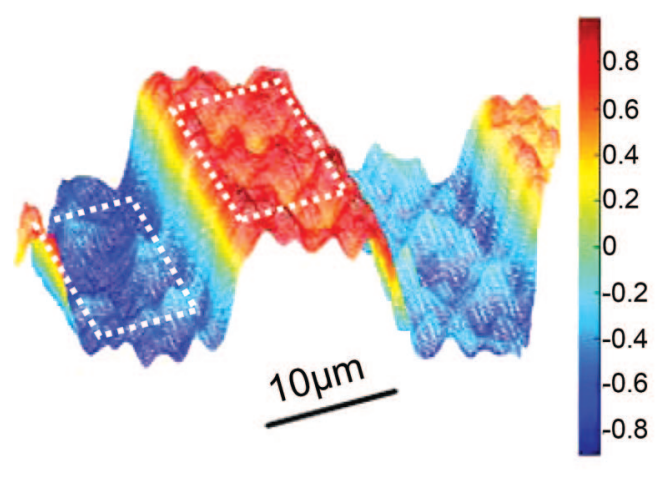

(c)

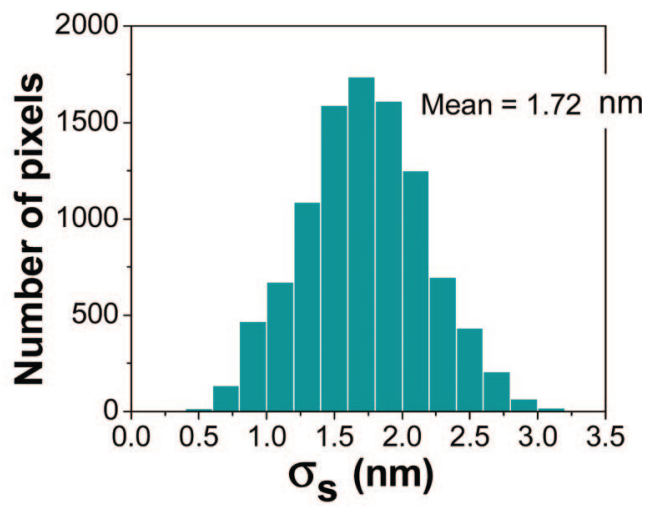

(b)

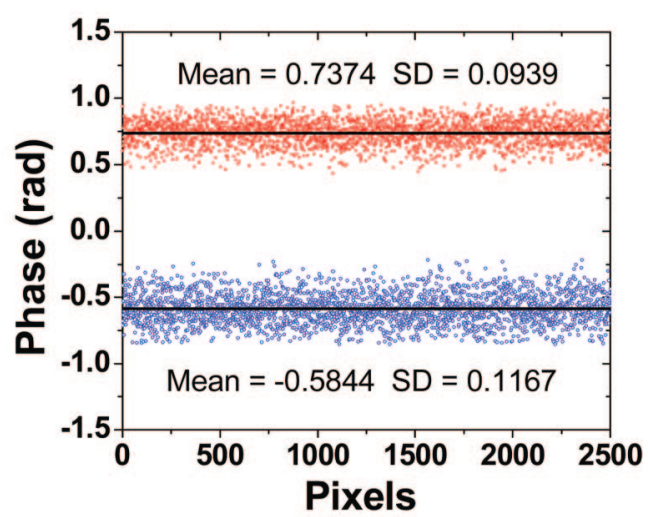

(d)

Fig. 3. (Color online) (a) Quantitative phase image of background. The shaded bar indicates the phase in radians. The limited field of view is to enlarge the diffraction spot at the Fourier plane of the dc component. (b) Histogram of temporal path-length standard deviations associated with pixels within the rectangular region shown in (a). (c) Profile of the phase grating obtained using the f-FPM. The shaded bar indicates the phase in radians. (d) Phase values measured in each pixel of the two rectangular regions shown in (c). 
The factor $\beta$ is obtained by using the procedure described in Ref. 21. The resulting phase $\phi$ is unwrapped if necessary, and the resulting phase images are filtered with a median filter over an area that corresponds to the optical diffraction limit, i.e., $3 \times$ 3 pixels $(\sim 500 \mathrm{~nm})$.

\section{Fast Fourier Phase Microscope Phase Stability and Accuracy}

To assess the temporal stability of the system, we captured quantitative phase images of the background, i.e., field of view with no sample. Sets of quantitative phase images were recorded over $3 \mathrm{~s}$ at $100 \mathrm{~ms}$ /image. Figure 3(a) shows an example of such a background image. The phase fluctuations associated with the $100 \times 100$ pixel region shown in Fig. 3 (a) were analyzed in terms of the standard deviation for each pixel. Each phase value is easily translated into optical length $s$, as $s=\phi \lambda /(2 \pi)$. The resulting histogram of the path-length standard deviations is shown in Fig. 3(b). The mean of the histogram has a value of $1.7 \mathrm{~nm}$. This remarkably low value of phase noise is attributable largely to the common path geometry of our interferometric system.

To demonstrate the accuracy of the measurements, we acquired the phase image of a phase grating characterized by a rectangular glass profile of height $571 \pm 10 \mathrm{~nm}$, as measured by stylus profilometry. Figure 3(c) shows the quantitative phase profile of the grating immersed in water. Figure $3(\mathrm{~d})$ shows the distribution of phase values in each pixel of the regions indicated at the top and bottom of the grating. The depth of the grating profile is determined from the average phase difference between these top and bottom regions and using the refractive indices of the glass (1.52) and water (1.33). The resulting average value for the measured height is $589 \mathrm{~nm}$, which agrees very well with the profilometry measurement. The measured standard deviation of the phase difference between the two regions is approximately 100 mrad, which is mainly attributable to the spatial nonuniformities of the grating surface and the impurities in the glass.

\section{Fast Fourier Phase Microscope Cell Imaging and Digital Processing}

We used the f-FPM to image live cells in culture. Live human epithelial (HeLa) cells were grown in the Dulbecco's Modified Eagle Medium containing 10\% fetal calf serum. These cells were imaged directly in culture conditions without additional preparation. Figure 4(a) shows a f-FPM image of a live HeLa cell. Unlike conventional phase microscopy (e.g., phase contrast and DIC), which can reveal cell structures qualitatively, the f-FPM provides a quantitative map of the cell in terms of optical path-length shift. Thus in Fig. 4(a) the nucleus of the cell can be easily recognized in the central region of the cell that is characterized by a higher phase shift. However, since the optical path length is integrated along the beam propagation direction, the sharpness of subcellular structures tends to

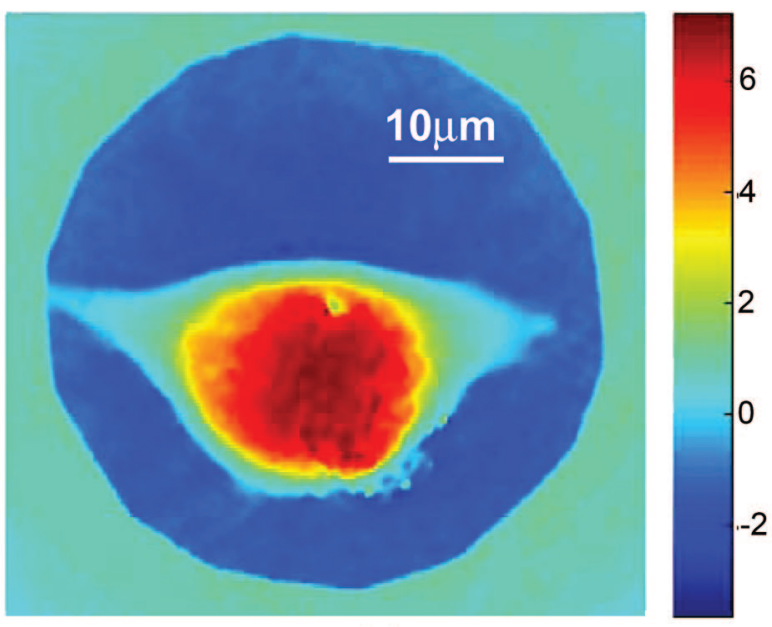

(a)

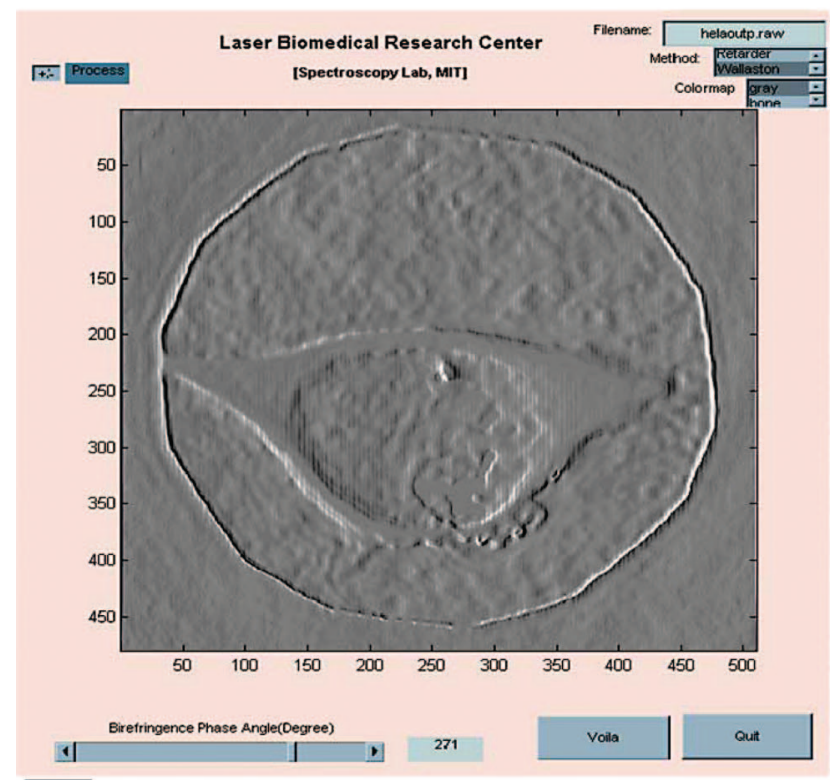

(b)

Fig. 4. (Color online) (a) f-FPM image of a live HeLa cell. (b) Digital DIC image obtained from the image in (a). The visual interface for numerical processing is also shown.

decrease. DIC microscopy, on the other hand, produces high contrast images of cells and organelles because it is sensitive to phase gradients rather than the phase itself. Using this idea, we numerically processed the quantitative phase image to implement what we refer to as digital DIC. This operation is possible because of the knowledge of the quantitative phase map provided by the f-FPM. Before computing its spatial gradient, the quantitative phase image is filtered numerically to reduce the contribution of the medium spatialfrequency range. We convert the phase image to an intensity map by computing the cosine of the phase, which is what would be measured if the sample field were interfered with a plane-wave reference. To provide flexibility in adjusting contrast, we apply a variable phase shift to the argument of this cosine function. Finally, we take the gradient of this intensity 

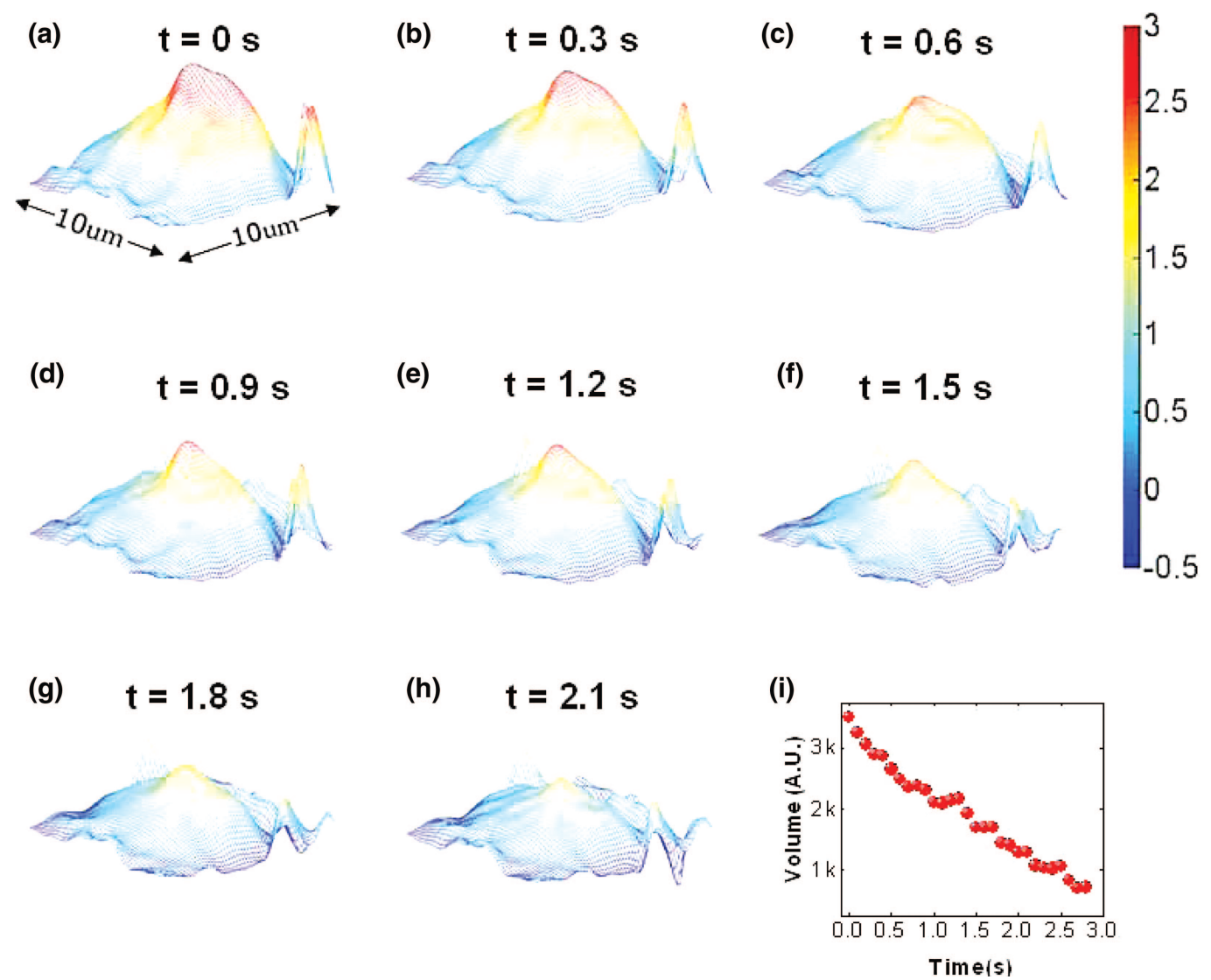

Fig. 5. (Color online) (a)-(h) Series of f-FPM images of a dissolving sugar crystal. The shaded bar indicates the phase in radians. (i) Temporal evolution of the volume enclosed by the surface $\phi(x, y)$.

image. Figure 4(b) displays the digital DIC image of a HeLa cell with an addition phase shift by $89^{\circ}$. As can be seen, the cell and nucleus boundaries are clearly visible with higher contrast than in the quantitative phase image. The graphic interface provides a slidebar control phase shift, which can be viewed as the digital analog to the Wollaston prism in typical DIC microscopes. With this numerical processing, a single f-FPM measurement can provide complementary views of the same sample without sample preparation, which is particularly appealing as the contrast is intrinsically generated.

\section{Quantitative Investigation of Dynamic Phenomena}

To demonstrate the near-video-rate imaging capability of the f-FPM, we continuously acquired quantitative phase images of dissolving sugar crystals in water. The dissolving rate of the sugar was adjusted by controlling a heating coil that surrounded the sample. Figure 5 shows a series of quantitative phase images of a dissolving sugar crystal. As can be seen, the dynamic change in crystal thickness and volume can be quantitatively monitored. With knowledge of the crystal refractive index, the evolution of the thickness can be inferred quantitatively. Of course, this technique can be applied to studying other dynamic phenomena in transparent systems, such as crystal growth, that are very difficult to monitor otherwise.

\section{Summary and Conclusions}

Owing to its remarkable stability, the fast Fourier phase microscope presented here may become a useful tool for biomedical research. The increased acquisition speed allows applications such as cell membrane fluctuations. The f-FPM has the appealing feature that it can be interfaced with an existing optical microscope. We anticipate that the f-FPM can become an empowering add on to a typical microscope. It is important to note the similarity between Fourier phase microscopy and diffraction phase microscopy ${ }^{23}$ (DPM) developed in our laboratory. Both techniques use the unscattered light transmitted through the sample (dc component) as a reference for a common-path interferometer, and thus they are both very stable. However, Fourier phase microscopy and DPM geometries differ significantly in several aspects. First, DPM is a single-shot technique, which is amenable to fast acquisition rates. However, DPM requires high-temporal-coherence illumination, which makes it susceptible to fringes from spurious reflections. On the other hand, Fourier phase microscopy can be implemented with low-coherence light (see Ref. 21), which eliminates specular patterns. Nevertheless, Fourier phase microscopy requires the acquisition of four different interferograms for each resulting phase image. Thus one can decide on the best choice between Fourier phase microscopy and DPM depending on the specific application targeted. 
This research was carried out at the Laser Biomedical Research Center, G. R. Harrison Laboratory, Massachusetts Institute of Technology, and was partially supported by National Institutes of Health grant P41 RR 02594 and by Hamamatsu Photonics K.K.

\section{References}

1. F. Zernike, "How I discovered phase contrast," Science 121, 345-349 (1955).

2. F. H. Smith, "Microscopic interferometry," Research (London) 8, 385-395 (1955)

3. D. Huang, E. A. Swanson, C. P. Lin, J. S. Schuman, W. G. Stinson, W. Chang, M. R. Hee, T. Flotte, K. Gregory, C. A. Puliafito, and J. G. Fujimoto, "Optical coherence tomography," Science 254, 1178-1181 (1991)

4. C. G. Rylander, D. P. Dave, T. Akkin, T. E. Milner, K. R. Diller, and A. J. Welch, "Quantitative phase-contrast imaging of cells with phase-sensitive optical coherence microscopy," Opt. Lett. 29, 1509-1511 (2004).

5. T. Akkin, D. P. Dave, T. E. Milner, and H. G. Rylander, "Detection of neural activity using phase-sensitive optical lowcoherence reflectometry," Opt. Express 12, 2377-2386 (2004).

6. C. Fang-Yen, M. C. Chu, H. S. Seung, R. R. Dasari, and M. S. Feld, "Noncontact measurement of nerve displacement during action potential with a dual-beam low-coherence interferometer," Opt. Lett. 29, 2028-2030 (2004).

7. M. A. Choma, A. K. Ellerbee, C. H. Yang, T. L. Creazzo, and J. A. Izatt, "Spectral-domain phase microscopy," Opt. Lett. 30, 1162-1164 (2005).

8. C. H. Yang, A. Wax, R. R. Dasari, and M. S. Feld, "Phasedispersion optical tomography," Opt. Lett. 26, 686-688 (2001)

9. C. H. Yang, A. Wax, I. Georgakoudi, E. B. Hanlon, K. Badizadegan, R. R. Dasari, and M. S. Feld, "Interferometric phasedispersion microscopy," Opt. Lett. 25, 1526-1528 (2000).

10. C. Yang, A. Wax, M. S. Hahn, K. Badizadegan, R. R. Dasari, and M. S. Feld, "Phase-referenced interferometer with subwavelength and subhertz sensitivity applied to the study of cell membrane dynamics, Opt. Lett. 26, 1271-1273 (2001).

11. G. A. Dunn and D. Zicha, eds., Using DRIMAPS System of Transmission Interference Microscopy to Study Cell Behavior (Academic, 1997)

12. D. Zicha and G. A. Dunn, "An image-processing system for cell behavior studies in subconfluent cultures," J. Microsc. 179, 11-21 (1995).

13. D. Zicha, E. Genot, G. A. Dunn, and I. M. Kramer, "TGF beta
1 induces a cell-cycle-dependent increase in motility of epithelial cells,” J. Cell Sci. 112, 447-454 (1999).

14. T. E. Gureyev, A. Roberts, and K. A. Nugent, "Phase retrieval with the transport-of-intensity equation-matrix solution with use of Zernike polynomials," J. Opt. Soc. Am. A 12, 1932-1941 (1995).

15. T. E. Gureyev, A. Roberts, and K. A. Nugent, "Partially coherent fields, the transport-of-intensity equation and phase uniqueness," J. Opt. Soc. Am. A 12, 1942-1946 (1995).

16. D. Gabor, "A new microscopic principle," Nature 161, 777-778 (1948).

17. T. Zhang and I. Yamaguchi, "Three-dimensional microscopy with phase-shifting digital holography," Opt. Lett. 23, 12211223 (1998).

18. P. Marquet, B. Rappaz, P. J. Magistretti, E. Cuche, Y. Emery, T. Colomb, and C. Depeursinge, "Digital holographic microscopy: a noninvasive contrast imaging technique allowing quantitative visualization of living cells with subwavelength axial accuracy," Opt. Lett. 30, 468-470 (2005).

19. B. Rappaz, P. Marquet, E. Cuche, Y. Emery, C. Depeursinge, and P. J. Magistretti, "Measurement of the integral refractive index and dynamic cell morphometry of living cells with digital holographic microscopy," Opt. Express 13, 9361-9373 (2005).

20. F. Charriere, A. Marian, F. Montfort, J. Kuehn, T. Colomb, E Cuche, P. Marquet, and C. Depeursinge, "Cell refractive index tomography by digital holographic microscopy,” Opt. Lett. 31, 178-180 (2006)

21. G. Popescu, L. P. Deflores, J. C. Vaughan, K. Badizadegan, H. Iwai, R. R. Dasari, and M. S. Feld, "Fourier phase microscopy for investigation of biological structures and dynamics," Opt. Lett. 29, 2503-2505 (2004).

22. T. Ikeda, G. Popescu, R. R. Dasari, and M. S. Feld, "Hilbert phase microscopy for investigating fast dynamics in transparent systems," Opt. Lett. 30, 1165-1168 (2005).

23. G. Popescu, T. Ikeda, R. R. Dasari, and M. S. Feld, "Diffraction phase microscopy for quantifying cell structure and dynamics," Opt. Lett. 31, 775-777 (2006).

24. G. Popescu, T. Ikeda, C. A. Best, K. Badizadegan, R. R. Dasari, and M. S. Feld, "Erythrocyte structure and dynamics quantified by Hilbert phase microscopy," J. Biomed. Opt. Lett. 10, 060503 (2005)

25. H. Kadono, M. Ogusu, and S. Toyooka, "Phase-shifting common-path interferometer using a liquid-crystal modulator," Opt. Commun. 110, 391-400 (1994).

26. J. Gluckstad and P. C. Mogensen, "Optimal phase contrast in common-path interferometry," Appl. Opt. 40, 268-282 (2001). 